Background Eosinophilic oesophagitis (EOE) is a chronic immune-mediated inflammatory disease of the oesophagus, characterised by symptoms of oesophageal dysfunction and eosinophilic infiltration at oesophageal biopsy. Despite a growing body of research surrounding EOE, our understanding of its natural history and optimal management remains limited. To shed more light on this, the clinical characteristics, diagnosis and management of patients diagnosed with EOE presenting to our tertiary Paediatric Gastroenterology unit were studied.

Methods Case notes and electronic records of children diagnosed with EOE were reviewed retrospectively from a twoyear period (July 2018 to July 2020). Children were eligible for inclusion if they had 1) histological confirmation of the presence of $>15$ eosinophils per high power field in at least one oesophageal mucosal biopsy taken at endoscopy; and 2) been discussed in the Paediatric Gastroenterology MDT for clinical-pathological correlation. Children with concomitant inflammatory diseases were excluded. Data on presenting features, investigations, management and response to treatment were collated and analysed using Microsoft Excel (Version 16.43).

Results A total of 28 children with a diagnosis of EOE fulfilled inclusion criteria for this study, 20 (71.4\%) males with a mean (SD) age of 8.91 (4.22) years. A quarter of children were referred by general practitioners and the remainder by hospital paediatricians and surgeons. Symptoms of dyspepsia and dysphagia predominated among $64.3 \%$ and $46.4 \%$ of subjects respectively. For $55.6 \%$ of children an allergic condition was also present, most commonly food allergy (42.9\%) (figure 1). Blood eosinophils were elevated in $78.6 \%$ of children (mean (SD) $0.64(0.43)$ ). Twenty-four (88.9\%) children had been started on a proton-pump inhibitor (PPI) prior to initial endoscopy and $63.6 \%$ were PPI-responsive. Macroscopic

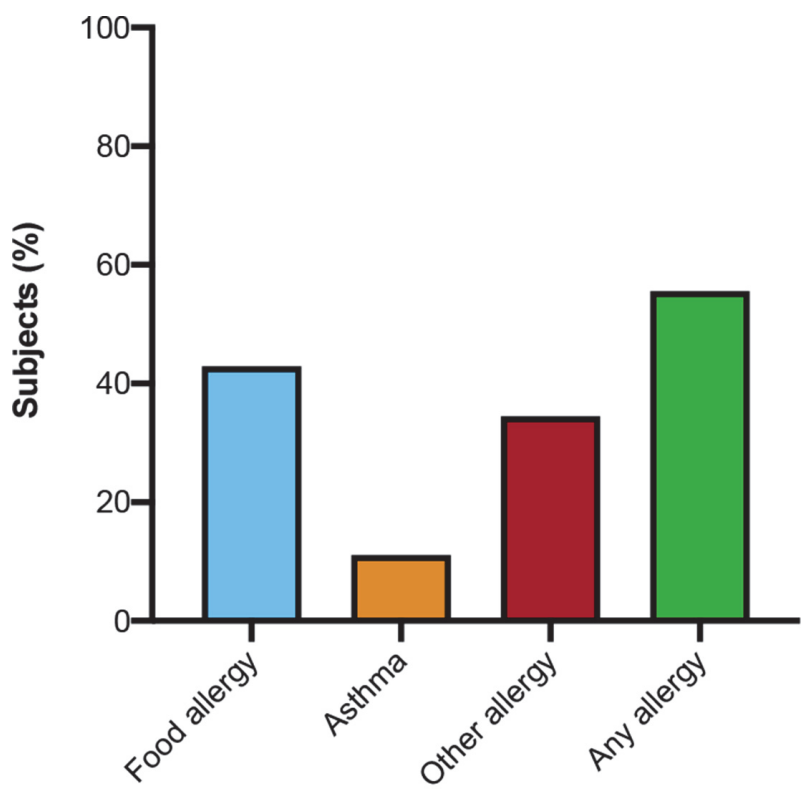

Atopic risk factors

Abstract P12 Figure 1 Prevalence of different types of allergies among children with EOE diagnosed at Leicester Royal Infirmary Other allergies $=$ subjects with allergic rhinitis or eczema. Any allergy = subjects with at least one of either food allergy, asthma or other allergy. features of EOE were evident in $65.4 \%$ of subjects. Upon diagnosis, 24 children (85.7\%) were reviewed by a dietician and started on an elimination diet (75\% empiric versus $25 \%$ testing-directed). At subsequent review, $83.3 \%$ had clinically improved although only $52.9 \%$ showed histological evidence of improvement at reassessment. Mean (SD) time between initial endoscopy and reassessment was 10.5 (8.39) months. A further 12 children (28.6\%) went on to have at least one more endoscopy. Topical steroid treatment with fluticasone was reserved as a second line treatment and given to three children (10.7\%). Limitations to this study include the retrospective design, limiting comparison of treatment approaches and evaluation of longer-term outcomes among children with EOE.

Conclusion In a cohort of children with EOE diagnosed at Leicester Royal Infirmary, male sex predominated whilst symptoms of dyspepsia or dysphagia, a history of atopy and elevated blood eosinophils were highly prevalent. Over $80 \%$ of children showed a clinical improvement with PPI therapy and dietary measures alone, highlighting the benefit of robust dietetic support. However, clinical improvement did not always correlate with endoscopic improvement. Moreover, escalation to second-line steroid treatment was uncommon, in part due to local challenges procuring orodispersible budesonide, which is not licensed in children.

\section{P13 CLINICAL FEATURES SIGNIFICANTLY ASSOCIATED WITH HIGHER RISK OF CATHETER-RELATED BLOOD STREAM INFECTION (CRBSI) IN CHILDREN ON LONG-TERM PARENTERAL NUTRITION (PN)}

${ }^{1}$ Maria Giovanna Puoti, ${ }^{2}$ Chiara D'Eusebio, 'Zafar Zaidi, ${ }^{1}$ Hannah Littlechild, ${ }^{1}$ Emily King, ${ }^{1}$ Jutta Koglmeier, Susan Hill. ${ }^{1}$ Great Ormond Street Hospital; ${ }^{2}$ University Hospital of Turin

\subsection{6/flgastro-2021-bspghan.24}

Objectives and study Catheter-related bloodstream infection (CRBSI) is a common and serious complication of parenteral nutrition (PN). We aimed to determine incidence and risk factors for CRBSI in children receiving long-term home $\mathrm{PN}$ at home for Intestinal Failure (IF).

Methods Diagnosis of CRBSI was based on clinical manifestations of infection such as fever, rigors, and/or hypotension together with positive blood culture obtained via the central venous catheter (CVC) and absence of other potential sources of infection. The incidence of CRBSI was measured as number of catheter-related episodes per 1000 catheter days. Data regarding potential risk factors for CRBSI such as young age $<5$ years, oral/enteral feeding, enteral tube feeding, enterocutaneous stoma, number of CVC lumens, absence of ileocecal valve (ICV), number of $\mathrm{PN}$ and lipid infusions/week were recorded.

Results The study group included 58 children (26 male, aged 7.2 \pm 4.6 years). Aetiology of IF included gastro-intestinal motility disorder (26/58), short bowel syndrome (SBS) (21/ $58)$ and enteropathy (Ent) (11/58). There were a total of 58414 catheter observation-days. Thirty-one of 58 (53.4\%) children (15 M, aged 5.8 \pm 4.3 years) were diagnosed with 108 CRBSIs, a rate of $1.85 / 1000$ catheter days. The median (range) number of CRBSI episodes per patient was 1 (0-14). The median days receiving PN was 1391 (range 75-1565), the median weekly PN infusion frequency was 7 days (range 1-7) and the median weekly lipid infusion was 3 days (range 
$0-5)$. CRBSI patients had significantly more PN infusions/ week (7, range $2-7$ vs 7 , range $1-7 ; \mathrm{P}<0.0001)$, presence of enterocutaneous stoma $(71.3 \%$ vs $27 \%$; $\mathrm{P}<0.0001)$, double-lumen CVC $(24.1 \%$ vs $4.8 \%$; $\mathrm{P}<0.0001)$, tube feeding $(38.9 \%$ vs $23.8 \% ; \mathrm{P}<0.05)$ and less oral/enteral feeding $(53.7 . \%$ vs $77.8 . \%$; P $<0.0005)$ compared to patients with no episodes of CRBSI.

Motility disorder group showed highest risk of CRBSI (HR 2.1; [95\% CI 1.84-3.21]; P <0.00001), total episodes of CRBSI $(61.3 \%$ vs SBS $31.8 \%$ and Ent $27.6 \%$; $<<0.0001)$ and CRBSI rate/1000 catheter days (motility disorder 2.7/1000, SBS $1.17 / 1000$, Ent $1 / 1000 ; \mathrm{P}<0.0001)$.

Log-rank survival analysis showed that frequency of $\mathrm{PN}$ infusion/week (HR 1.3; [95\% CI 1.10-.1.51]; P <0.0001), enterocutaneous stoma (HR 3.9; [95\% CI 1.95-7.76]; P $<0.0001$ ), absence of ICV (HR 2.37; [95\% CI 1.17-4.81]; $\mathrm{P}<0.05$ ), double-lumen CVC (HR 2.51; [95\% CI 1.703.86]; $\mathrm{P}<0.01$ ), age under 5 years (HR 2.26; [95\% CI 2.16-3.39]; $\mathrm{P}<0.000001)$ and male sex (HR 2.51; [95\% CI 1.64-3.86]; $\mathrm{P}<0.00001)$ were significantly associated with higher CRBSI rate. Conversely oral/enteral feeding significantly reduced the risk of CRBSI (HR 0.54; [95\% CI 0.47-0.98.]; $\mathrm{P}<0.001)$. COX multivariate analysis showed that only enterocutaneous stoma, age $<5$ years and doublelumen CVC were independently associated with a higher risk of CRBSI.

Conclusion Almost half of the children receiving home PN for IF do not develop CRBSI. Children with motility disorder are at highest risk of CRBSI. Moreover, the presence of enterocutaneous stoma, double-lumen $\mathrm{CVC}$ and age $<5$ years significantly increase the risk of developing CRBSI. These risk factors should be considered in the management of home PN in children with IF.

\section{P14 CLINICAL GENOMICS FOR THE DIAGNOSIS OF MONOGENIC FORMS OF INFLAMMATORY BOWEL DISEASE: THE 2020 ESPGHAN POSITION PAPER AND ITS IMPLICATIONS FOR UK SERVICE PROVISION IN 2021}

${ }^{1}$ Holm H Uhlig, ${ }^{2}$ David Wilson, ${ }^{3}$ Jochen Kammermeier, ${ }^{4}$ Fevronia Kiparissi, ${ }^{4}$ Kimberly Gilmour, ${ }^{2}$ Richard Russell. ${ }^{1}$ University Oxford; ${ }^{2}$ University of Edinburgh; ${ }^{3}$ Evelina Hospital; ${ }^{4}$ Great Ormond Street Hospital

\subsection{6/flgastro-2021-bspghan.25}

Background It is important to identify patients with monogenic IBD since management including response to biologics and surgery plus the role of stem cell transplantation may differ from classical IBD. We report on the 2020 Position paper of the PORTO group of ESPGHAN for the use of genomics to diagnose monogenic causes of IBD.

Methods Paediatric IBD specialists from the Paediatric IBD Porto group of the European Society of Paediatric Gastroenterology, Hepatology and Nutrition (ESPGHAN) and specialists from several monogenic IBD research consortia reached a consensus of standard of care. Our systematic literature review covered indications, technologies (targeted panel, exome and genome sequencing), gene panel setup, cost-effectiveness of genetic screening, and requirements for the clinical care setting.

Results Next-generation DNA sequencing technologies are recommended to diagnose monogenic causes of IBD in routine clinical practice, embedded in the setting of multidisciplinary patient care. Routine genetic screening is not recommended for all IBD patients but instead genetic testing should be considered in the context of age of IBD onset (infantile IBD, very early onset IBD, paediatric or young adult IBD) and on further key criteria such as family history, relevant comorbidities and extraintestinal manifestations. Genetic testing is also recommended in advance of hematopoietic stem cell transplantation. We present a diagnostic algorithm that includes a gene panel of seventy-five monogenic IBD genes. We discuss how these recommendations can be implemented from 2021 onwards into the UK NHS health care system. Lastly, we present a UK-focused health care utilisation pathway highlighting the available UK clinical resources, clinical targeted panel sequencing and exome sequencing strategies in the UK, and regional immune validation pathways.

Summary Genomic technologies should be considered an integral part of patient care to investigate patients at risk for monogenic forms of IBD in the UK.

\section{P16 DIAGNOSIS AND MANAGEMENT OF FUNCTIONAL GASTROINTESTINAL DISORDERS: A CLINICAL AUDIT}

Aimee Staunton*, ${ }^{1}$ Amelia Kataria Golestaneh*, ${ }^{2}$ Stephen Allen, ${ }^{2}$ Manjula Velayudhan Nair. ' University of Liverpool School of Medicine; ${ }^{2}$ Alder Hey Children's NHS Foundation Trust, Liverpool, UK; * Joint first authors

\subsection{6/flgastro-2021-bspghan.26}

Background Functional gastrointestinal disorders (FGIDs), now understood as disorders of gut-brain interactions, are a group of recurring gastrointestinal symptoms which after appropriate medical evaluation cannot be attributed to another medical condition. Clinical evaluation and management remain challenging. The recently updated Rome IV criteria are symptombased guidelines enabling classification and prevalence estimates of childhood FGIDs.

Aims To determine the prevalence of FGIDs amongst children presenting to Alder Hey gastroenterology outpatient clinics according to Rome IV criteria. To compare Rome IV with clinical diagnoses and describe clinical management.

Methods The referral letters and case records of all children attending gastroenterology clinics in January 2019 were

Abstract P16 Table 1 Diagnoses according to Rome IV criteria and clinical assessment and clinical management

\begin{tabular}{|c|c|c|c|c|}
\hline \multicolumn{2}{|c|}{$\begin{array}{l}\text { Rome IV diagnosis } \\
\mathrm{N} \text { definite/probable (\%) }\end{array}$} & \multicolumn{2}{|c|}{ Clinical diagnosis $\mathrm{N}(\%)$} & Clinical management $\mathrm{N}$ \\
\hline \multirow[t]{8}{*}{ IBS } & $\begin{array}{l}24 / 3 \\
(42.9)\end{array}$ & IBS & $\begin{array}{l}11 \\
(40.7)\end{array}$ & $\begin{array}{l}\text { Dietary review/ } \\
\text { modification } 11\end{array}$ \\
\hline & & Abdominal pain & $\begin{array}{l}6 \\
(22.2)\end{array}$ & $\begin{array}{l}\text { Anti-spasmodic } 8 \\
\text { Stool softener/laxative } 8\end{array}$ \\
\hline & & Functional pain & $\begin{array}{l}3 \\
(11.1)\end{array}$ & $\begin{array}{l}\text { PPI } 4 \\
\text { Symptom/stool diary } 2\end{array}$ \\
\hline & & $\begin{array}{l}\text { Non-specific } \\
\text { symptoms }\end{array}$ & $2(7.4)$ & $\begin{array}{l}\text { Psychological therapy/ } \\
\text { review } 2\end{array}$ \\
\hline & & Cyclic vomiting & $1(3.7)$ & Threadworm eradication \\
\hline & & Cow's milk allergy & $1(3.7)$ & 2 \\
\hline & & $\begin{array}{l}\text { Gastritis/ } \\
\text { constipation }\end{array}$ & $1(3.7)$ & $\begin{array}{l}\text { Anti-diarrhoeal } 2 \\
\text { Probiotic } 1\end{array}$ \\
\hline & & None given & $2(7.4)$ & \\
\hline
\end{tabular}

